\title{
15 Years of R\&D on High Field Accelerator Magnets at FNAL
}

\author{
Emanuela Barzi ${ }^{\text {a, }}$, Alexander V. Zlobin ${ }^{\mathrm{a}}$ \\ ${ }^{a}$ Fermi National Accelerator Laboratory (FNAL), Batavia, IL, USA
}

\begin{abstract}
The High Field Magnet (HFM) Program at Fermi National Accelerator Laboratory (FNAL) has been developing $\mathrm{Nb}_{3} \mathrm{Sn}_{\text {superconducting }}$ magnets, materials and technologies for present and future particle accelerators since the late 1990s. This paper summarizes the main results of the $\mathrm{Nb}_{3} \mathrm{Sn}$ accelerator magnet and superconductor R\&D at FNAL and outlines the Program next steps.
\end{abstract}

Keywords: accelerator magnet, composite wire, dipole, $\mathrm{Nb}_{3} \mathrm{Sn}$, quadrupole, Rutherford cable

\section{Introduction}

For a circular collider of given size, its energy is limited by the strength of the bending dipole magnets. Moreover, for both linear and circular machines, their maximum luminosity is determined, among other factors, by the strength of quadrupoles used for the final beam focusing. That is why there has been enduring interest in the High Energy Physics (HEP) and Particle Accelerator communities in higher-field and higher-gradient accelerator magnets [1]. The highest fields have been achieved using superconducting electromagnets. The nominal field of $\mathrm{NbTi}$ dipole magnets used in all present high energy machines, including the Large Hadron Collider (LHC) at the European Organization for Nuclear Research (CERN), is limited to 8 to $9 \mathrm{~T}$ at an operating temperature of $1.9 \mathrm{~K} \mathrm{[2].}$

To raise the magnetic field in accelerator magnets beyond the limit of NbTi magnets, superconductors with higher critical parameters are needed. Among the many known high field superconductors only $\mathrm{Nb}_{3} \mathrm{Sn}, \mathrm{Nb}_{3} \mathrm{Al}, \mathrm{Bi}-2212\left(\mathrm{Bi}_{2} \mathrm{Sr}_{2} \mathrm{CaCu}_{2} \mathrm{O}_{8}\right)$ and $\mathrm{REBCO}\left(\mathrm{REBa}_{2} \mathrm{Cu}_{3} \mathrm{O}_{7}\right.$, where $\mathrm{RE}$ stands for rare earth) are sufficiently developed to be presently used in magnets above $10 \mathrm{~T}$ [3], [4]. However, only $\mathrm{Nb}_{3} \mathrm{Sn}, \mathrm{Nb}_{3} \mathrm{Al}$, and $\mathrm{Bi}-2212$ superconductors are industrially produced in the form of composite wires in long $(\sim 1 \mathrm{~km})$ length, as required for accelerator magnets.

Accelerator magnets need high current multi-strand superconducting cables to reduce the number of turns in the coils, and thus magnet inductance. The Rutherford cable, developed at the Rutherford Appleton Laboratory (RAL) [5], has played a crucial role in establishing NbTi accelerator magnet technology. It is widely used in modern high energy accelerators thanks to its excellent mechanical, electrical and thermal properties.

During the past decade, FNAL, home of the first superconducting accelerator (the Tevatron), has been developing new $\mathrm{Nb}_{3} \mathrm{Sn}$ accelerator magnet technologies in the framework of the High Field Magnet (HFM) program. $\mathrm{Nb}_{3} \mathrm{Sn}$ accelerator magnets can provide operating fields up to $15 \mathrm{~T}$ and significantly increase the coil temperature margin. Such magnets are being developed for the LHC IR upgrade, Muon Collider Storage Ring,

*Corresponding author

Email address: barzi@fnal.gov and present and future high energy hadron colliders. The program began in 1998 with the development of small-aperture arc dipoles for the very Large Hadron Collider (VLHC) [6]. In 2005, the emphasis of the program was shifted toward large-aperture $\mathrm{Nb}_{3} \mathrm{Sn}$ quadrupoles for an LHC IR upgrade [7]. From 2011 to 2015 FNAL has been developing in collaboration with CERN an $11 \mathrm{~T}$ dipole for the LHC collimation system upgrade [8]. Since 2015 the program has been redirected towards $15 \mathrm{~T}$ or higher field magnets for a future circular collider [9].

The HFM R\&D program includes the development of basic technologies and studies of main magnet parameters (maximum field, quench performance, field quality) and their reproducibility using a series of short models, and then proceeds with the demonstration of technology scale-up using relatively long coils. Along the way, the HFM program has made several breakthroughs in $\mathrm{Nb}_{3} \mathrm{Sn}$ accelerator magnet technology. The most important include the development and demonstration of high performance $\mathrm{Nb}_{3} \mathrm{Sn}$ strands and cables, a reliable and reproducible coil fabrication technology, and a variety of accelerator quality mechanical structures and coil pre-load techniques [10]. The status and the main results of the $\mathrm{Nb}_{3} \mathrm{Sn}$ accelerator magnet R\&D at FNAL are summarized in this paper.

\section{Strands and Cables R\&D}

Cross sections of composite $\mathrm{Nb}_{3} \mathrm{Sn}, \mathrm{Nb}_{3} \mathrm{Al}$ and $\mathrm{Bi}-2212$ wires are shown in Fig. 1. Their actual potential for high field accelerator magnets is being extensively studied.

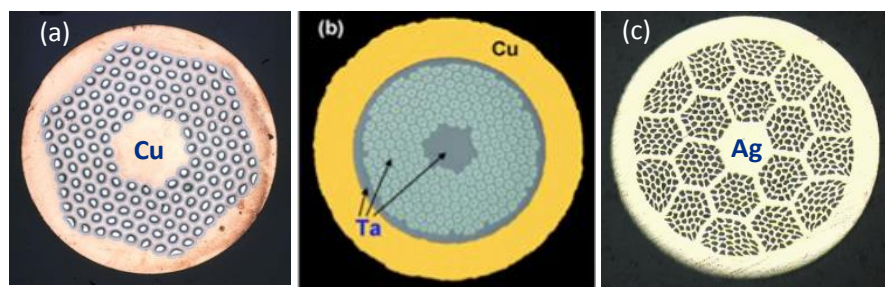

Fig. 1. Cross sections of $\mathrm{Nb}_{3} \mathrm{Sn}$ (left), $\mathrm{Nb}_{3} \mathrm{Al}$ (center) and $\mathrm{Bi}-2212$ (right) composite wires. Courtesy of OST (USA) and NIMS (Japan).

The critical current density $J_{c}$ of $\mathrm{Nb}_{3} \mathrm{Sn}$ more than doubled between 1998 and 2007 thanks to the US Conductor Development 
Program. As a result, the $J_{c}$ at $12 \mathrm{~T}$ of $\mathrm{Nb}_{3} \mathrm{Sn}$ wires exceeds the $J_{c}$ at $5 \mathrm{~T}$ of $\mathrm{NbTi}$ wires. However, a higher $J_{c}$ at $15 \mathrm{~T}$ and lower costs are desired for $\mathrm{Nb}_{3} \mathrm{Sn}$. A large progress was made in Japan for $\mathrm{Nb}_{3} \mathrm{Al}$ composite wire production and performance thanks to the Rapid-Heating Quenching Transformation process. $\mathrm{Nb}_{3} \mathrm{Al}$ has lower strain sensitivity than $\mathrm{Nb}_{3} \mathrm{Sn}$ and thus potential for operation at higher forces. Nevertheless, there are still significant fabrication challenges to be solved to improve $J_{c}$ and make $\mathrm{Nb}_{3} \mathrm{Al}$ cost competitive with $\mathrm{Nb}_{3} \mathrm{Sn}$. Heat treating Bi-2212 under high pressure increases the wire $J_{c}$. However, this result has to be demonstrated for multi-strand cables and coils in order to be useful for accelerator magnets.

$\mathrm{Nb}_{3} \mathrm{Sn}$ composite wires are currently produced using three main methods: bronze, internal tin (IT), and powder-in-tube (PIT) [4]. At present the IT Restacked-Rod Process (RRP®) by Oxford Superconducting Technology (OST) and PIT by Bruker-EAS are the only two wires with sufficiently high $J_{c}$ for HEP applications that are available in large quantities from industry. FNAL's main contributions to high field $\mathrm{Nb}_{3} \mathrm{Sn}$ conductor advances include the development and optimization with industry of composite wires with improved stability problem due to flux jumps and low sensitivity to transverse plastic deformations. These results are widely recognized, and the wires were adopted by CERN and by the LHC Accelerator Research Program (LARP). FNAL also developed with NIMS, KEK and Japanese industry a $\mathrm{Nb}_{3} \mathrm{Al}$ wire and Rutherford cable that established the use of this conductor in magnets for the first time [11]. In addition, FNAL has been performing development and optimization of Rutherford cables based on Bi-2212 wires with the goal of demonstrating this superconductor for use in high field accelerator magnets.

Examples of Rutherford cable based on $\mathrm{Nb}_{3} \mathrm{Sn}, \mathrm{Nb}_{3} \mathrm{Al}$ and $\mathrm{Bi}$ 2212 strands are shown in Fig. 2. Cable development is performed by designing and fabricating samples of different geometries using state-of-the-art wires, with the purpose of studying the effect of cable parameters and processing on its performance. This includes for instance the sensitivity of electrical properties and internal structure to cable compaction, measurements of cable stability and AC losses, measurements of 3D cable expansion during the fabrication process and during reaction, etc. FNAL's main contributions to high field cable advances include the development of challenging $\mathrm{Nb}_{3} \mathrm{Sn}$ cables with high aspect ratios and with an inner stainless steel core that were successfully used in the $11 \mathrm{~T}$ dipole prototypes for CERN.

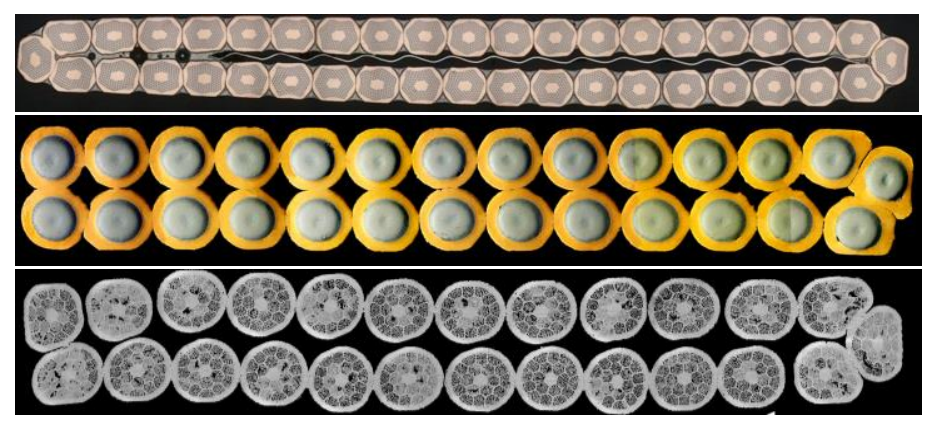

Fig. 2. Cross sections of keystoned 40 -strand $(0.7 \mathrm{~mm}) \mathrm{Nb}_{3}$ Sn Rutherford cable with an $11 \mathrm{~mm}$ wide and $25 \mu \mathrm{m}$ thick stainless steel core (top) [8]; 27-strand $(1 \mathrm{~mm}) \mathrm{Nb}_{3} \mathrm{Al}$ rectangular cable (middle) [11]; 25-strand $(0.8 \mathrm{~mm}) \mathrm{Bi}-2212$ rectangular cable (bottom) [12].

\section{Magnet R\&D}

\subsection{T Dipoles for VLHC}

The design and main parameters of FNAL dipole models of the HFDA series are described in [13]. These magnets have been developed as baseline dipoles for the VLHC, which was extensively studied in the US in 2000 [5]. The cross section of the dipole cold mass is shown in Fig. 3 (left). This magnet was designed to provide a nominal field of 10 to $11 \mathrm{~T}\left(B_{\max } \sim 12 \mathrm{~T}\right)$ in a $43.5 \mathrm{~mm}$ aperture at an operating temperature of $4.5 \mathrm{~K}$. The main R\&D goal of this model magnet series was to develop a robust $\mathrm{Nb}_{3} \mathrm{Sn}$ coil technology based on the Wind\&React (W\&R) approach and an inexpensive mechanical structure suitable for industrialization. The magnet design was based on a two-layer shell-type coil and a cold iron yoke. To reduce the magnet cost, a compact collarless mechanical structure with Al clamps, a $400 \mathrm{~mm}$ iron yoke and a $10 \mathrm{~mm}$ stainless steel skin were used.
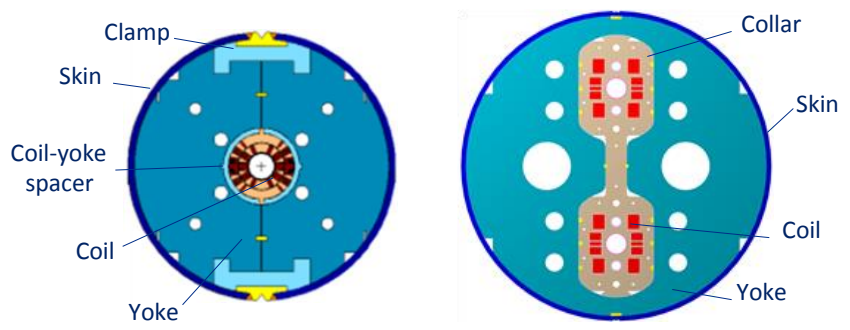

Fig. 3. Cross section of single-aperture HFDA dipole (left) and twin-aperture common coil HFDC dipole (right).

FNAL also developed a common coil dipole design (HFDC), which meets the VLHC requirements and allows using the React\&Wind (R\&W) technology [13]. The main features of this magnet are a wide 60 -strand cable based on high $J_{c} 0.7 \mathrm{~mm} \mathrm{Nb}_{3} \mathrm{Sn}$ strand, a single-layer coil confined in an innovative collar structure with two $40 \mathrm{~mm}$ bores and a cold, vertically-split iron yoke. The HFDC dipole cross section is shown in Fig. 3 (right). The goals of this work were to study the possibilities and limitations of the R\&W technology and develop a $10 \mathrm{~T} \mathrm{Nb}_{3} \mathrm{Sn}$ accelerator quality common coil dipole based on this approach. An advantage of the $\mathrm{R} \& \mathrm{~W}$ method is the possibility of using traditional materials developed for NbTi accelerator magnets in the past 30 years.

\subsection{0-mm aperture Quadrupoles for LHC}

The design and parameters of FNAL's quadrupole models of the TQC series are described in [10]. These magnets were proposed and used as a technological model of a new generation of large-aperture IR quadrupoles for the planned LHC luminosity upgrade. The TQC cross section is shown in Fig. 4. This model magnet series was designed to provide the same nominal field gradient of $200 \mathrm{~T} / \mathrm{m}\left(G_{\max } \sim 250 \mathrm{~T} / \mathrm{m}\right)$ in a $90-\mathrm{mm}$ aperture at the same operating temperature of $1.9 \mathrm{~K}$ as the present $70-\mathrm{mm} \mathrm{NbTi}$ LHC IR quadrupoles (MQXB). The quadrupole design consists of a two-layer shell-type coil and a cold iron yoke. The design and technology of quadrupole coils used in TQC models largely rested on the results of the HFDA dipole program described above. The TQC mechanical structure is based on a slightly modified mechanical structure of the present LHC MQXB. It includes a 25- 
mm thick stainless steel collar, a 400-mm iron yoke and a $12 \mathrm{~mm}$ thick stainless steel skin.

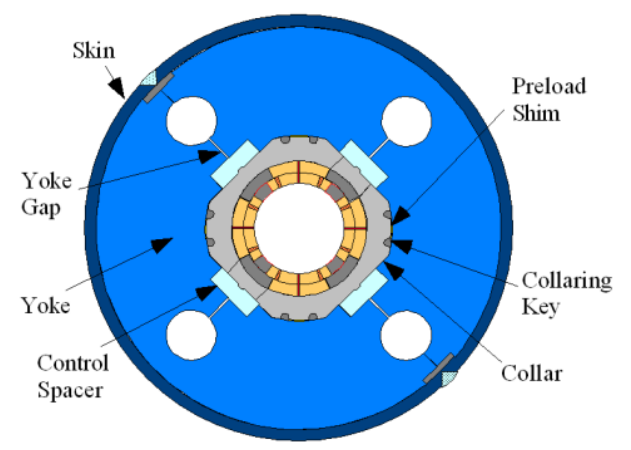

Fig. 4. TQC quadrupole cross section.

\subsection{T Dipoles for LHC Collimation System Upgrade}

Additional collimators are planned in the LHC Dispersion Suppression areas around points 2, 3, 7, and at the CMS and ATLAS detectors [7]. Creating room in the ring for these collimators requires replacing a number of $8.33 \mathrm{~T} 15 \mathrm{~m}$ long $\mathrm{NbTi}$ main dipoles $(\mathrm{MB})$ with shorter $11 \mathrm{~T} \mathrm{Nb}_{3} \mathrm{Sn}$ dipoles $(\mathrm{MBH})$. The latter need to be compatible with the LHC lattice and main systems and deliver the same integrated bending strength at the LHC nominal operation current.

Design concepts of the $11 \mathrm{~T} \mathrm{Nb}_{3} \mathrm{Sn}$ dipole developed at FNAL in collaboration with CERN for LHC upgrades in both singleaperture and twin-aperture configurations are described in [8], [14]. The dipole design features 2-layer shell-type $\mathrm{Nb}_{3} \mathrm{Sn}$ coils, separate stainless steel collars for each aperture and a MB yoke which was modified in the area of the collar yoke interface. The magnet coil was designed to provide a dipole field of $11 \mathrm{~T}$ in a $60 \mathrm{~mm}$ aperture at the LHC nominal operation current of $11.85 \mathrm{kA}$ with $20 \%$ margin along the magnet load line. The chosen coil aperture of $60 \mathrm{~mm}$ is slightly larger than the MB dipole aperture, which avoids bending the $\mathrm{Nb}_{3} \mathrm{Sn}$ coils to accommodate the LHC beam sagitta. Fig. 6 shows the cross sections of the $11 \mathrm{~T}$ dipole in both single-aperture and twinaperture configurations.

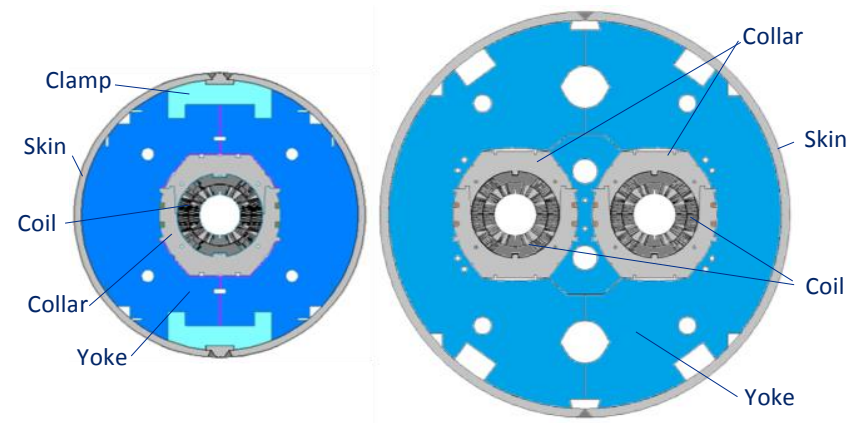

Fig. 5. Single-aperture MBHSP (left) and twin-aperture MBHDP (right) designs of the $11 \mathrm{~T}$ dipole.

\subsection{T Dipoles for a Future Circular Collider}

To build a $\sim 100 \mathrm{TeV}$ Hadron Collider (HC) in a $\sim 100 \mathrm{~km}$ tunnel, $\sim 15 \mathrm{~T}$ dipoles operating at 1.9 or $4.5 \mathrm{~K}$ with 15 to $20 \%$ margin are needed. A nominal operating field up to 15 to $16 \mathrm{~T}$ can be provided by the $\mathrm{Nb}_{3} \mathrm{Sn}$ technology. A practical demonstration of this field level in accelerator-quality magnets and a substantial reduction of magnet costs are key conditions for the realization of such a machine.

FNAL has started the development of a $15 \mathrm{~T} \mathrm{Nb}_{3} \mathrm{Sn}$ dipole demonstrator for a $100 \mathrm{TeV}$ scale $\mathrm{HC}$ based on the optimized "cos-theta" coil design [14]. The design concept of the $15 \mathrm{~T}$ dipole demonstrator cold mass is shown in Fig. 6 [16]. The 4-layer graded coil is supported by a vertically split iron yoke, aluminum clamps, and a thick stainless steel skin. The coil assembly, surrounded by a $2 \mathrm{~mm}$ stainless steel spacer, is placed in between the two half-yokes and braced with two clamps. The skin and the clamps are pre-tensioned under a press to provide an initial coil pre-stress at room temperature. The axial Lorentz forces on the coil ends are intercepted by two thick end plates connected by eight tie rods running through dedicated holes in the iron yoke.

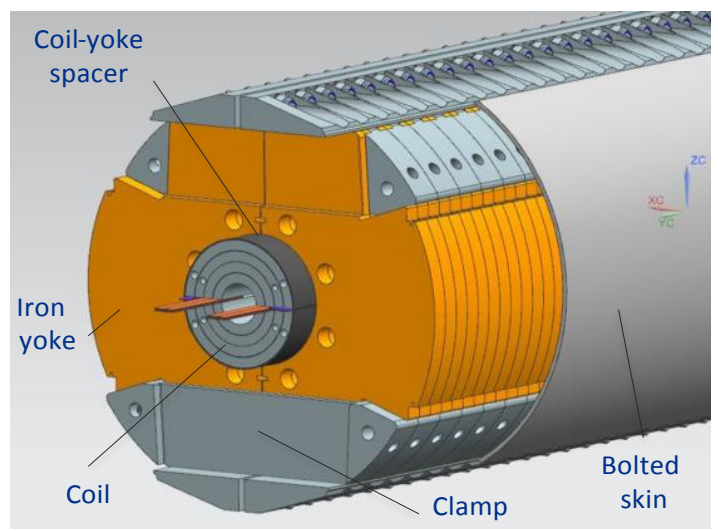

Fig. 6. Cold mass design of the $15 \mathrm{~T}$ dipole demonstrator.

\subsection{Summary of Magnet Performance}

The magnet models were tested at FNAL in liquid helium at $4.5 \mathrm{~K}, 1.9 \mathrm{~K}$ and intermediate temperatures. Fig. 7 shows the maximum field reached in the FNAL $\mathrm{Nb}_{3} \mathrm{Sn}$ dipole and quadrupole models that were herein described, as well as in special coil test structures (CTS), also called "mirror configurations", where only one coil is tested and the others are replaced by special iron blocks.

HFDA and HFDC models. Six short HFDA dipoles and six HFDM dipole CTSs were built and tested from 2002 to 2006. This was the first series of nearly identical $\mathrm{Nb}_{3} \mathrm{Sn}$ accelerator magnets that provided the first data on magnet quench performance and field quality and their reproducibility. The first three dipole models, HFDA02-04, made of IT Modified Jelly Roll (MJR) strand with relatively large superconducting subelement size, were limited by flux jumps in the superconductor and reached only 5 to $6 \mathrm{~T}$ or 50 to $60 \%$ of their design field. The last three dipole models HFDA05-07, made of a more stable PIT strand with smaller filament size, reached $B_{\max }=9.4 \mathrm{~T}$ at $4.5 \mathrm{~K}$, and $10.2 \mathrm{~T}$ at $2.2 \mathrm{~K}$, i.e. $100 \%$ of magnet short sample limit (SSL) at both temperatures. The maximum field reached in these models was limited by the relatively low critical current density of the PIT strand. Nevertheless, these models clearly demonstrated that the developed $\mathrm{Nb}_{3} \mathrm{Sn}$ coil technology and magnet mechanical 
structure are adequate for $10 \mathrm{~T}$ accelerator magnets. A dipole coil made of high $J_{c}$ RRP-108/127 strand and tested in 2006 using the dipole test structure HFDM06 reached $B_{\max }=11.4 \mathrm{~T}$ at $4.5 \mathrm{~K}$ ( $97 \%$ of $S S L$ ), confirming robustness of the developed dipole coil technology and mechanical structure.

Three 1-m long racetracks and one common coil dipole model HFDC 01 based on reacted $\mathrm{Nb}_{3} \mathrm{Sn}$ cables were fabricated and tested from 2001 to 2003. All magnets survived the complicated fabrication process but reached only 60 to $75 \%$ of the expected short sample limit.

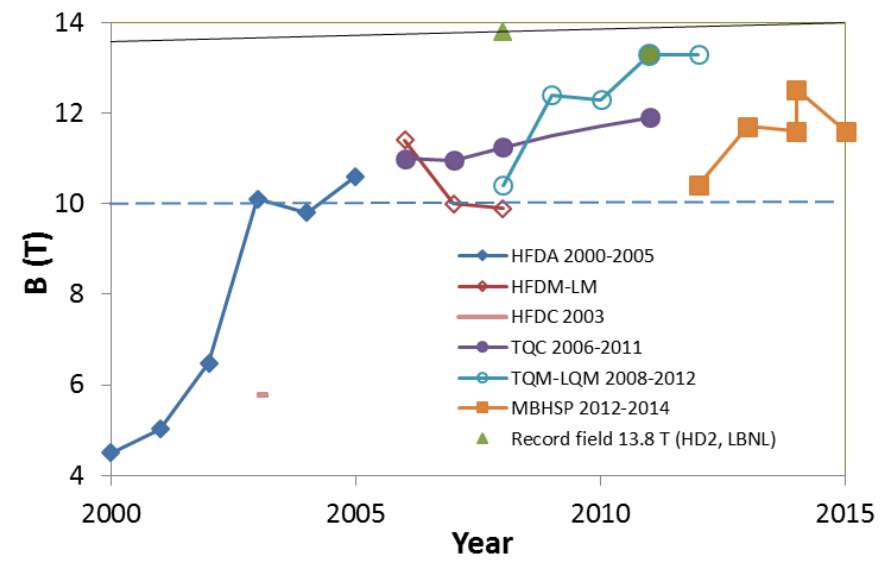

Fig. 7. Maximum field reached in the FNAL dipole and quadrupole models (solid markers) and mirror configurations (open markers).

TQC model series. From 2007 to 2010 seven 90-mm aperture TQC quadrupole models and six TQM quadrupole CTS were fabricated and tested, expanding and enriching the previous results and experience. The first TQC quadrupole models TQC01a and TQC01b, made of the low $J_{c}$ MJR strand, reached the nominal design field gradient of $200 \mathrm{~T} / \mathrm{m}$ at $1.9 \mathrm{~K}$. One TQC model TQC02Ea was collared using traditional quadrupole collars and the multi-pass partial compression technique, whereas TQC02Eb was collared using dipole-style collars. The quench performance of the quadrupole models collared using different collaring schemes was similar. The maximum field gradient reached at $4.5 \mathrm{~K}$ in TQC models based on high $J_{c}$ RRP-54/61 strand was $211 \mathrm{~T} / \mathrm{m}$ or $\sim 90 \%$ of magnet $S S L$. At $3 \mathrm{~K}$ it increased to $217 \mathrm{~T} / \mathrm{m}$. However, at lower temperatures it reduced to $\sim 200 \mathrm{~T} / \mathrm{m}$ due to flux jumps in the superconducting strands. Eventually, a TQC quadrupole model with coils made of RRP$108 / 127$ wire achieved the design field gradient of $220 \mathrm{~T} / \mathrm{m}$ at the nominal operation temperature of $1.9 \mathrm{~K}$.

MBHSP and MBHDP models. A 2-m long single-aperture $\mathrm{Nb}_{3} \mathrm{Sn}$ dipole demonstrator was fabricated and tested at FNAL in June 2012. Then, to improve the magnet design and performance, and demonstrate performance reproducibility, seven 1-m long coils were fabricated from 2012 to 2014. Of these, four were assembled and collared in dipoles and tested in a single-aperture configuration first. Both single-aperture dipole models were trained to $11.6 \mathrm{~T}$ at $1.9 \mathrm{~K}$, or $97 \%$ of the dipole design field of $12 \mathrm{~T}$. The two tested 1-m long collared dipoles have then been assembled in the first twin-aperture dipole model and successfully tested at FNAL in February 2014 reaching $11.5 \mathrm{~T}$ at $1.9 \mathrm{~K}$.
$15 T$ Dipole demonstrator. The design of a $15 \mathrm{~T}$ dipole demonstrator is in progress. Inner and outer cable samples have been fabricated and tested showing low degradation. Procurement of coil parts and tooling has started. Magnet assembly and first test are planned for 2016.

The quench performance and field quality data confirm the good reproducibility and robustness of the developed $\mathrm{Nb}_{3} \mathrm{Sn}$ magnet designs and technology. The scale up program of the $\mathrm{Nb}_{3} \mathrm{Sn}$ accelerator magnet technology was successfully performed at FNAL by testing 2-m and 4-m long dipole and quadrupole coils in their corresponding mirror structures.

\section{Conclusions}

The FNAL High Field Magnet Program has been developing $\mathrm{Nb}_{3} \mathrm{Sn}$ superconducting magnets, materials and technologies for present and future particle accelerators since the late 1990s. Its most important breakthroughs include: the development and demonstration of high-performance $\mathrm{Nb}_{3} \mathrm{Sn}, \mathrm{Nb}_{3} \mathrm{Al}$ and $\mathrm{Bi}-2212$ strands and cables; reliable and reproducible short and long coil technologies ready for production; accelerator quality mechanical structures; the first world series of $\mathrm{Nb}_{3} \mathrm{Sn}$ dipole and quadrupole models with reproducible magnet parameters; innovative field quality correction techniques; etc. These advances make it possible to consider 10 to $12 \mathrm{~T} \mathrm{Nb}_{3} \mathrm{Sn}$ magnets in accelerators, specifically for the planned LHC luminosity upgrades. The Program is presently exploring limits of the $\mathrm{Nb}_{3} \mathrm{Sn}$ magnet technology by developing a $15 \mathrm{~T} \mathrm{Nb}_{3} \mathrm{Sn}$ dipole demonstrator. The ultimate goal is the development of magnet technologies based on both High and Low Temperature Superconductors to achieve fields of $20 \mathrm{~T}$ and beyond.

\section{Acknowledgment}

This work was supported by Fermi Research Alliance, LLC, under contract No. DE-AC02-07CH11359 with the US Department of Energy.

[1] A. Devred, S.A. Gourlay, A. Yamamoto, IEEE Trans. on App. Supercond., v. 15 , no. 2, p. 1192 (2005).

[2] L. Rossi, IEEE Trans. on Appl. Supercond., v. 14, no. 2, p. 153 (2004).

[3] H. Kumakura, Japanese Journal of Appl. Physics, 51, p. 010003 (2012).

[4] L. Bottura and A. Godeke, Rev. of Accel. Science and Technology, 5, 25 (2012).

[5] G. Gallagher-Daggit, Rutherford Laboratory Memorandum No. RHEL/M/A25, 1973).

[6] "Design study for a staged Very Large Hadron Collider", Fermilab-TM2149, June 4, 2001.

[7] L. Bottura et al., IEEE Trans. on Appl. Supercond., v. 22, no. 3, 4002008 (2012).

[8] M. Karppinen et al., IEEE Trans. on Appl. Supercond., v. 22, no. 3, 4901504 (2012).

[9] F. Zimmermann et al., Proc. of IPAC2014, Dresden, Germany, 2014, p. 1.

[10] A.V. Zlobin, "Status of $\mathrm{Nb}_{3} \mathrm{Sn}$ Accelerator Magnet R\&D at Fermilab", CERN Yellow Report CERN-2011-003, p. 50 [arXiv:1108.1869].

[11] R. Yamada et al., IEEE Trans. on Appl. Supercond., v. 17, no. 2, p. 1461 (2007).

[12] E. Barzi et al., AIP Conf. Proc. 986, 431 (2008).

[13] A.V. Zlobin et al., IEEE Trans. on Appl. Supercond., v. 15, no. 2, p. 1113 (2005).

[14] A.V. Zlobin et al., Proc. of PAC2011, NYC, 2011, p. 1460.

[15] A.V. Zlobin et al., Proc. of IPAC2015, Richmond, VA, 2015, p. 3365

[16] I. Novitski et al., IEEE Trans. on Appl. Supercond., v. 26, no.2, 2016. 